\title{
MICROMECHANICAL SIMULATION OF A MARTENSITIC TRANSFORMATION BY FINITE ELEMENTS
}

\author{
J.F. GANGHOFFER, S. DENIS, E. GAUTIER, A. SIMON, K. SIMONSSON*and S. SJÖSTRÖM* \\ Laboratoire de Science et Génie des Matériaux Métalliques, Ecole des Mines, Parc de Saurupt, \\ F-54042 Nancy cedex, France \\ *Linköping Institute of Technology, S-58183 Linköping, Sweden
}

\begin{abstract}
A micromechanical model describing the martensitic transformation on the grain scale has been developed, using Finite Elements. First results gained from the simulation illustrate how the morphological evolution within the grain is directly controlled by the internal stress state. The reversible and irreversible part of transformation "plasticity" strain and their evolution with the transformation can then be obtained from these calculations.
\end{abstract}

\section{1- Introduction}

A material undergoing a phase transformation and submitted to an external applied stress even lower than the yield stress of the weaker phase exhibits transformation "plasticity" deformation. For martensitic transformation, the so called transformation plasticity deformation is the result of two mechanisms : i) preferential orientation of the martensite plates by the stress and ii) anisotropic plastic accommodation of the transformation strains (shear strain and volumic variations) [1-4]. The first contribution which is correlated to the distribution of plate orientations is reversible when the reverse transformation will be produced and is characteristic of the shape memory effect; the second one is permanent. These deformations have been mostly correlated to the external applied stress. However since the large transformation strain associated with martensitic transformation must be mechanically accommodated, the transformation process will create high internal stresses in the surrounding of the new phase. In fact, the orientation of the habit plane will be controlled by the local stress anistropy and the characteristic lattice orientation within the grain .The Finite Element model developed here aims at a better understanding of the rather complex mechanical behaviour of a material undergoing a martensitic transformation. Particularly, the effect of internal stresses on kinetics and anisotropy of plate orientation will be investigated. A first attempt to differentiate between the strain contributions of the two basic mechanisms underlying transformation "plasticity" will be shown.

\section{2 - Description of the micromechanical model}

The micromechanical concepts and the description of martensitic transformation have been discussed in [5]. We recall here the essential features of the model. The transformation strain associated to the transformation can be described as an invariant plane strain, [6,7]. It consists in a shear $\gamma_{0}$ along an undistorted plane, the so-called habit plane, and a dilatation $\varepsilon_{0}$ normal to it. The strain components $\gamma_{0}$ and $\varepsilon_{0}$ of the transformation strain are often quite large : typical values for a ferrous alloy are respectively 0.19 and 0.03 [1].

The basic idea in our analysis is to assume that the material consists of identical representative cells, stacked in a repetitive way, which behave in the same way, simultaneously. From a micromechanical simulation of such a cell, the macroscopic behaviour (at the scale of a specimen) can then be obtained. The need to describe the aforementioned couplings at a sufficiently fine scale leads to use a grain as "cell". Since the physical situation involved is probably that of an assembly of grains with different shapes, sizes, lattice orientations, it is therefore a quite complex aggregate to model in the general case. Therefore, as a first step, a two dimensional model has been developed, where the cell consists of just one quadratic grain. The modelling of the representative cell (unit cell) is done with finite elements, where each element of the cell 
represents one region filled with the material of either of the two phases. We assume that the mechanics of continuum media governs the behaviour of the phases. Tranformation of an element is simulated by giving it an initial strain, corresponding to the transformation strain, and new mechanical properties. The grain is built by an assembly of 324 triangular elements (fig.1), under plane stress assumption and the boundary of the model is constrained to stay rectangular. A more thorough discussion about the choice of the boundary conditions is presented in [5].

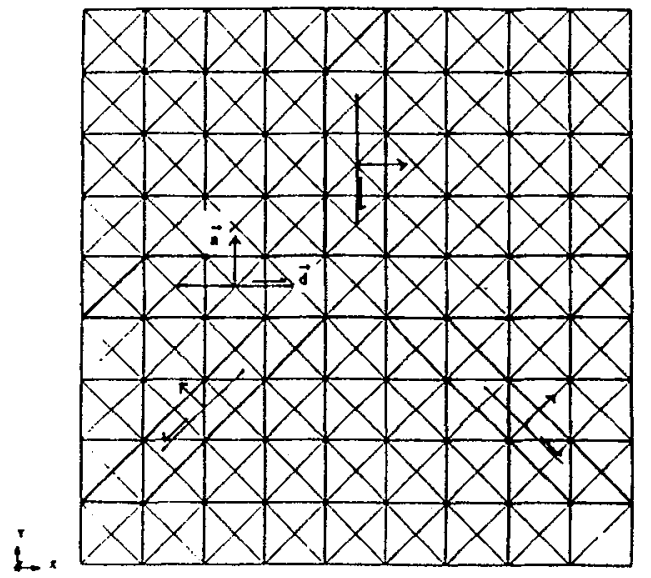

Figure 1: FE mesh and possible martensite plates

Considering the cristallography of the transformation, there is a discrete number of possible habit planes, for example 12 for ferrous alloys with $\{225\}$ habit planes. To simulate this, we have introduced 4 possible habit planes orientations in our 2 dimensional model, with a $45^{\circ}$ angle between each other. The choice of triangular elements allows effectively to associate these morphological features of plates with the mesh geometry. In each of these 4 directions, a plate can be constructed, which consists typically of a band of elements. (the illustration of possible plate pattern with their habit plane is shown on figure 1). The habit plane is parallel to the plate length. When such a plate transforms, all elements it consists of, receive the same transformation strain in a groupwise manner.

The order in which the plates transform, and their associated habit planes are determined in the following way : the local stress state in each untransformed element is projected onto each of the 4 possible normal directions (vector $\mathrm{n}$ on fig. 1). The stress state can then be reduced into a normal stress $\sigma_{\mathrm{n}}$ perpendicular to the habit plane and a shear stress $\tau$ along it (on direction $d$ on fig. 1). The element and orientation for which the mechanical driving force $\sigma_{n} \varepsilon_{0}+\tau \gamma_{0}$ (function of $n$ ) has the highest value will be the next nucleation point (for the next plate) and plate direction respectively. The plate stops when it reaches another already formed plate or the grain boundary. To simulate the actual morphology of a plate, we let it end with a sharp tip at the boundary.

The FE code used for the calculations is ASKA. The simulation starts with the application of an external load, which creates an initial stress state. Since all elements at this moment have the same mechanical driving force, a first nucleation point must be chosen and its habit plane is determined from the external load anisotropy. After that, the corresponding plate is found and an elastoplastic calculation is performed. After full incrementation of the transformation strain for this plate, all remaining untransformed elements will be checked whith regard to the previously described transformation criterion and a new start element for a plate will be determined. In this way, more and more plates will form until the whole grain is filled with the new phase. The input data are given in table 1. A schematic flowchart describing the main steps of the simulation is shown on fig. 2 below. 
TABLE 1

Input data for the FE calculation

\begin{tabular}{|c|c|c|c|c|}
\hline $\begin{array}{c}\text { Properties } \\
\text { Phase }\end{array}$ & $\begin{array}{c}\text { Young's modulus } \\
(\mathrm{MPa})\end{array}$ & Poisson's ratio & $\begin{array}{c}\text { Yield Strength } \\
(\mathrm{MPa})\end{array}$ & $\begin{array}{c}\text { Hardening siope } \\
(\mathrm{MPa})\end{array}$ \\
\hline AUSTENITE & $2.10^{5}$ & 0.3 & 300 & 3800 \\
\hline MARTENSITE & $2.10^{5}$ & 0.3 & 1000 & 8300 \\
\hline \multicolumn{4}{|c|}{ Transformation parameters $\gamma 0=0.19 ; \varepsilon 0=0.03$} \\
\hline
\end{tabular}

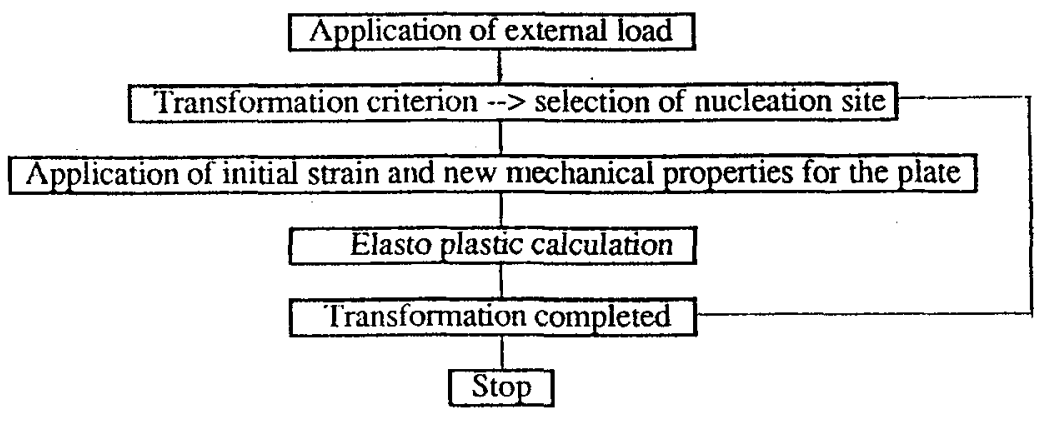

Figure 2: schematic flowchart of the simulation

\section{3 - Results and discussion}

From the FE calculation we get informations about the plate pattern in the grain, the local stress and strain states and the macroscopic deformation of the grain during the transformation. In this paper we have chosen to show only the plate pattern and the variations of the macroscopic deformation with the progress of transformation. The macroscopic total strain is defined as the displacement of one node of the edge perpendicular to the load direction. Moreover we have defined the reversible transformation strain as the accumulated contribution of the transformation strain component in the load direction $\left(\varepsilon_{x}{ }^{\text {tr }}\right)$ for each plate orientation multiplied by the fraction it represents. Figure 3 shows the components $\varepsilon_{\mathbf{x}}$ tr for the different possible habit plane orientations. This definition of a macroscopic transformation strain provides an evaluation of the stress-induced anisotropy of the deformation associated with the plate orientation distribution. The difference between the macroscopic total strain and the reversible transformation strain defines then the macroscopic resulting elastic and plastic strain. (Pure elastic calculations for a small external load evidence nearly identical values for total and transformation strains.)
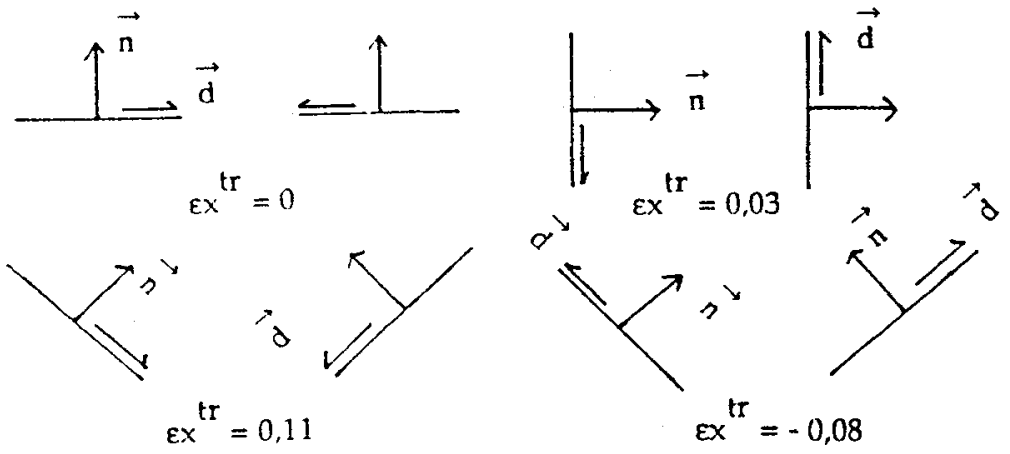

Figure 3 : different habit plane orientations ( $\mathrm{n}$ : normal, d: shear direction ) and associated transfonnation strain contribution $\left(\varepsilon_{x}{ }^{\text {tr }}\right)$ in the direction of the applied stress 
The first results of the simulation have evidenced two parameters, namely the size of the first plate and the magnitude of the applied load (only uniaxial cases are considered in this study), the effect of which we now analyse.

\section{3-1 Effect of the size of the first plate.}

Two calculations with a small applied stress (10MPa) have been performed, using different choices for the first transformed plate. The first one (fig.4a) is a long diagonal band while the second one is a much shorter band of the same orientation (figure $4 \mathrm{~b}$ ); they represent transformed fractions of about $10 \%$ and $3 \%$ respectively. The choice of a first long plate leads to a strong anisotropy of the plate pattern, most of the plates keeping that first orientation (fig. 4a). This tendency is reflected on the evolution of the transformation strain (fig. 5a), which keeps a high slope till about $30 \%$ transformed material (8 plates are formed), due mainly to the formation of plates 1 and 7 , each representing a fraction near $10 \%$. One observes that formation of plate $5\left(\varepsilon_{x}{ }^{\mathrm{tr}}=0.03\right)$ leads to the slope decrease at about $10 \%$. A saturation effect appears after $30 \%$, where non favorably oriented plates start to form (formation of plate 9 doesn't give any contribution of the transformation strain in the load direction). Plates still parallel to the first one form, but some with opposite shear direction, producing a set of twin related plates (e. g. plates $7,13,16,20)$. Such parallel disposition provides a quite physical way of accommodating the very large imposed shear strain along the plate length,and plastic flow will therefore be present mainly at the plate ends. This is further assessed by the nearly zero macroscopic plastic strain (fig. 5a) till about $70 \%$ transformation. At the end of the transformation, shorter plates with different orientations are built.Simultaneously,more plastic flow is observed (a more noticeable difference exists between the total and the transformation strain) due to the higher stresses generated.

A quite different plate pattern develops when starting with a first short band (fig. 4b). The immediately next forming plates $(2,4)$ receive other orientations and give rise to plastic strains in the load direction. The resulting pattern is less uniform, some bands keeping the orientation of the first (e.g. bands 6, 10, 17, 29, 35), alternating with horizontal and vertical bands (e.g. bands 2, 4, 11, 16, 25, 28, 37). The main contribution of such vertical bands occurs between about $50 \%$ and $70 \%$ transformed fractions, and a small positive plastic strain is measured during this interval (both opposite shear directions lead to a positive contribution of the strain in the load direction in that case). Such an effect is however quite complex to analyse and will need further investigation of the local results (stress states and distributions of plastic flow) of the calculations.

A related consequence of the plate pattern is the nearly linear evolution of the total strain. Such a plate pattern for a small load (relatively to the internal stresses created which are close to the yield stress of austenite [5]) validates the choice of a first short plate, which will therefore be kept for the next calculations.

\section{3-2 Effect of the magnitude of the applied load}

Results of the calculation with a $250 \mathrm{MPa}$ external load are presented on fig. $4 \mathrm{c}$ and $5 \mathrm{c}$. The macroscopic strain is extremely large in that case (up to $13 \%$ ), due to the cumulative contributions of favorably oriented plates. A difference with the run using $10 \mathrm{Mpa}$ (with first short plate) occurs from the very beginning, since the second plate is oblique for the higher load (it is vertical for $10 \mathrm{Mpa}$ ). Very few horizontal and vertical bands form in that case and most of the oblique bands (with either $45^{\circ}$ or $135^{\circ}$ orientation of their habit plane) present the same shear direction. Fewer twins are also observed. Applying a stress state near the austenite yield strength leads to internal stress levels of the same order of magnitude as the external load. The effect of internal stresses on the plate development is therefore much smaller as compared to the case with $10 \mathrm{MPa}$. Consequently applying $250 \mathrm{MPa}$ leads to a large deformation in the applied load direction all along the transformation since the principal directions of the local stress tensor are near that of the external one. This is further evidenced by the quite linear evolution of the transformation strain (fig. $5 \mathrm{c}$ ) in that case and by the occurence of large and more evenly distributed plastic flow from the beginning. Anisotropic plastic accommodation of transformation strains will be a large contribution to the total strain (about $50 \%$ ). Moreover, for the three presented calculations, the morphology of the plate (sharp tips) has large effects on the habit plane development, since many nucleation sites are found in the prolongation of previously formed plates due to the strong coupling of the plate end with the stiff boundary (high concentration of stresses and plastic flow are found at this place). Such effects will therefore be further investigated. 

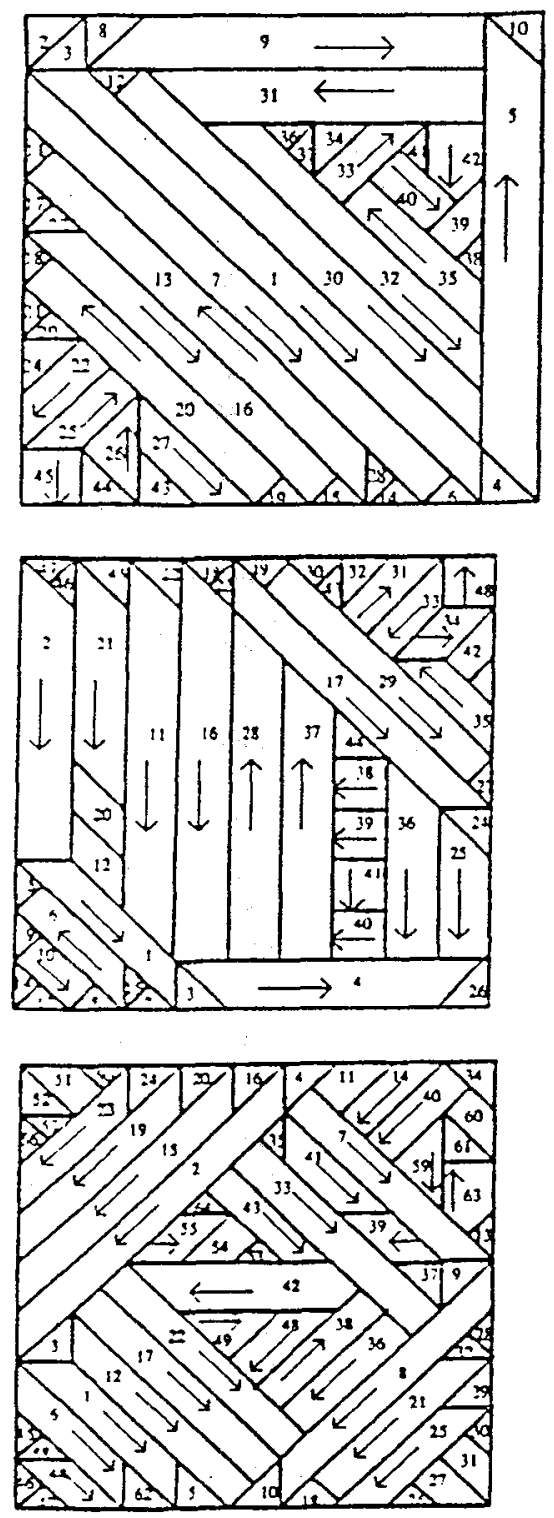

Figure 4: plate pattern evolution (order, orientation )

a ) First long plate / Sharp tips / $10 \mathrm{Mpa}$

b) First short plate / Sharp tips / $10 \mathrm{Mpa}$

c) First short plate / Sharp tips / $250 \mathrm{Mpa}$ a)

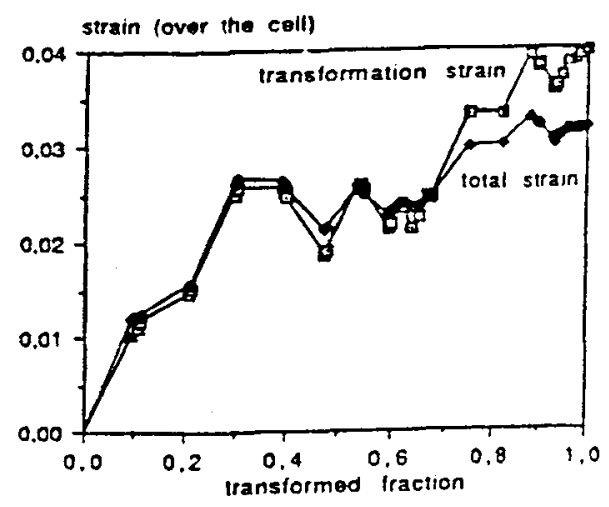

b)

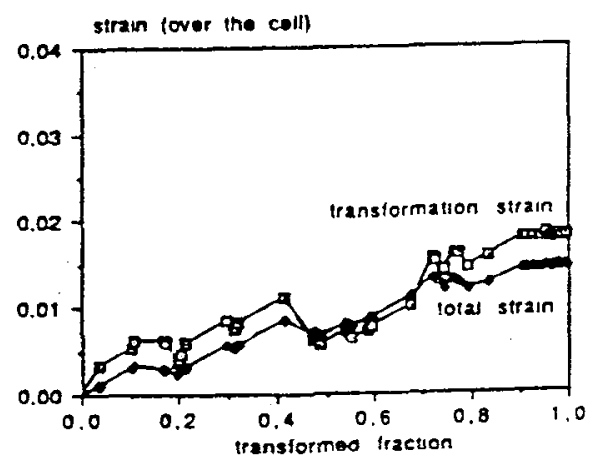

c)

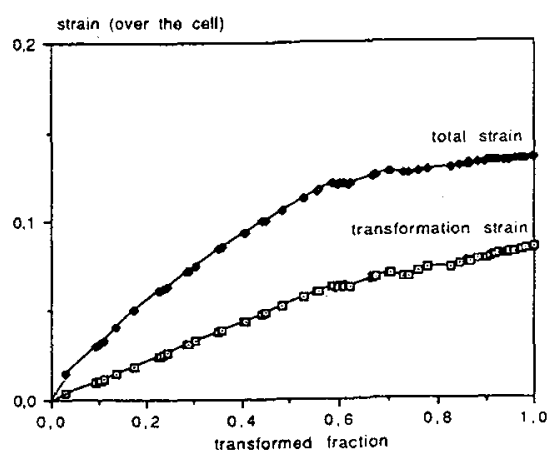

Figure 5 : evolutions of total and transformation strains with the transformed fraction in cases $a, b, c$ 


\section{4 - Conclusion}

The first results of our calculations show that the model allows to describe the distribution of plates orientation and the macroscopic deformation of a grain submitted to an external stress state. Moreover the two contributions to this strain, the reversible strain due to orientation of the martensite plates and the strain due to plastic accommodation can be analysed quantitatively for different external stresses when transformation progresses. This study is now going on in order to know more thoroughly the influence of the different parameters of the finite element model (size of the model, boundary conditions, size of the first plate...). Further calculations are needed for different applied stresses and different input data in order to put forward the way of variations of transformation "plasticity" deformations with the progress of the transformation, to compare them to experimental results and to discuss the validity of the plane stress model as to predict transformation "plasticity" deformation laws.

\section{Acknowledgments}

The work described has been supported jointly by CNRS (the French Center for Scientific Research) and STU (the Swedish Board for Technical Development). The computer calculations have been performed on a Cray XMP/48 at NSC (the National Supercomputer Centre, LINKÖPING, Sweden).

\section{References}

[1] E. GAUTIER, A. SIMON, G. BECK ,Proceedings International Conference on Strength of Metals and Alloys. Ed. H.J. Mc Queen,J.I. Dickson, J.J. Jonas and M.G. Akbens, 569, 1985.

[2] E. GAUTIER, A. SIMON, Proceedings Phase Transformations 87, The Institute of Metals ,Ed. G.W. LORIMER, 451, 1987.

[3] E. GAUTIER, X. M. ZHANG, A. SIMON, Proceedings International Conference on Residual Stresses (ICRS 2) Ed. G. Beck, S. Denis, A. Simon, Elsevier Applied Science, 777, 1989.

[4] S. DENIS, E. GAUTIER, A. SIMON, Proceedings International Conference on Residual Stresses (ICRS2) Ed. G. Beck, S. Denis, A. Simon, Elsevier Applied Science, 393, 1989.

[5] J.F. GANGHOFFER, K. SIMONSSON, S. DENIS, E. GAUTIER, S. SJÖSTRÖM, A. SIMON ICRS3, Tokushima (Japan) ,1991.

[6] M.S. WECHSLER, D.S. LIEBERMAN, T.A. READ, 1953 Trans. AIME, 197, 1503.

[7] E. PATOOR Thèse de Docteur, Ingénieur, Université de Metz, 1986. 